\title{
Evolutionary Dynamics of the Insurgency in Iraq: A Mathematical Model of the Battle for Hearts and Minds
}

\author{
JONATHAN DAVID FARLEY \\ Stanford University \\ Stanford, California, USA
}

\begin{abstract}
Individuals are not electrons. But in some situations, they behave very much alike. When it comes to many social and political questions, individuals' complex attitudes and beliefs must be boiled down to a simple yea ("spin up") or nay ("spin down"). Although human societies, like physical materials, are very complex, some of their properties can be determined by understanding the interactions that occur between individuals ("atoms"). In particular, the spread of a particular political opinion throughout a society can be modeled as the macroscopic manifestation of the myriad exchanges occurring at the local level between individuals and their neighbors. This model can be used to gain a qualitative foothold on the evolution of public opinion in Iraq for or against the U.S.-led occupation. It can also provide a mathematical instantiation of the "oil-spot" strategy proposed by Krepinevich (2005), which in turn could lead to a practical tool for commanders needing to allocate public relations resources in Iraq.
\end{abstract}

\section{PART I}

\section{Introduction}

How many men, women, and children must you win over to your cause before victory is assured? Conversely, how many men, women, and children in a society must turn against you before defeat is inevitable? Although intuition says that these numbers must be high, a certain model of public opinion based on ideas from statistical physics suggests that the answer may be smaller than one might at first think.

The following model has been proposed for understanding the spread of a political opinion throughout a society (Nowak, Szamrej, and Latané 1990, 371-372; Young 2004): View individuals like atoms in a magnetic material. Given a political question that an individual may either support or reject, view her as being +1 or "spin up" if she supports the issue, -1 or "spin down" if she rejects it. There is no "action at a distance": each individual

Received 14 November 2006; 5 January 2007.

Dr. Tony Harkin of the Division of Engineering and Applied Sciences at Harvard University made valuable contributions to Part II of this article. The author also thanks Dr. Mark Kayser of Nuffield College, University of Oxford for his comments regarding the article as well as Mr. David Patel of Stanford University for discussions about the "oil-spot" strategy.

Address correspondence to the author's current address: Jonathan David Farley, Department of Mathematics, California Institute of Technology, Pasadena, CA 91125, USA. E-mail: lattice.theory@gmail.com 
can only influence her immediate neighbors. The Ising model of statistical physics shows how local interactions can explain the behavior of a macroscopic body (Ising 1925; Peierls 1936; Cipra 1987). If two neighboring micromagnets are oriented in the same direction (both +1 or both -1 ), they tend to reinforce one another; if they are oriented in opposite directions (one +1 and the other-1), they tend to cancel. The material's overall magnetic orientation is determined by summing up all the $+1 \mathrm{~s}$ and $-1 \mathrm{~s}$.

Ising's discovery is that the physics underlying the local interactions between neighboring molecules is enough to explain the observed behavior of ferromagnetic materials: At normal temperatures the materials do not exhibit any particular magnetic properties (because every molecule has the same chance of being spin up as spin down, the summation is on the average very nearly 0 ), but there is a critical temperature below which all of the molecules suddenly align (either all +1 or all -1 ). This phenomenon is known as a phase transition. (Non-magnetic materials can also exhibit phase transitions, as, for instance, when water crystallizes into ice at the critical temperature $0^{\circ} \mathrm{C}$.)

What is interesting is that, under suitable conditions, human societies can also exhibit phase transitions. Suppose it is assumed that every individual has an opinion on a question $(+1$ or -1$)$ and that an individual's views can only be influenced by her neighbors. If society is evenly divided on an issue (e.g., abortion, social security reform, or Iraq) and the individuals holding view +1 are randomly distributed, the views of individuals may change, but, overall, society will remain evenly divided on the issue (there is no emergence of "public opinion"). If, however, the initial distribution of $+1 \mathrm{~s}$ is increased slightly (so that it is non-trivially above 50\% but still well below 100\%), and one allows for individuals to randomly switch opinions (an analogue of quantum fluctuation), it can happen that the +1 viewpoint sweeps across the society: public opinion crystallizes.

It is not argued that these hypotheses actually do hold for human societies (for instance, the initial distribution of $+1 \mathrm{~s}$ is rarely random; witness the "red state-blue state" divide in the United States). ${ }^{1}$ But the model, especially because of its simplicity, may shed some light on what is actually observed. In a final section the article gives some applications to the growth of anti-American sentiment in Iraq.

\section{The Simulation}

The article uses a probabilistic version of von Neumann's theory of cellular automata. (For aspects of the deterministic theory, see Wolfram 2002 and von Neumann 1966.) For $N$ a large integer, a two-dimensional torus viewed as an $N \times N$ grid is considered where one considers the cells in the top row to be adjacent to the cells in the bottom row, and similarly for the left and right columns. (This simulates the idea that New York and Los Angeles may be more closely connected than, say, New York and Tulsa.) For a fixed $p$ between 0 and 1 , the grid is randomly populated so that $p N^{2}$ of the cells are -1 . (For the sake of illustration, the +1 cells are colored red and the -1 cells are colored blue.) See Figures 1 and 2, a color-coded map of the United States by county, with red for Republican-leaning counties and blue for Democrat-leaning counties, a picture that bears a superficial similarity to Figure 1.

Each cell in the torus is influenced by its neighbors as follows: Add the values of a cell and its immediate neighbors to the north, south, east, and west. If the result is positive, the cell will be +1 in the next time interval; otherwise it will be -1 . See Figure 3 for an illustration of this rule for a $4 \times 4$ grid. $^{2}$

In the standard theory, the evolution of the cellular automaton is purely deterministic, although it may exhibit non-periodic and seemingly "random" behavior. Genuine 


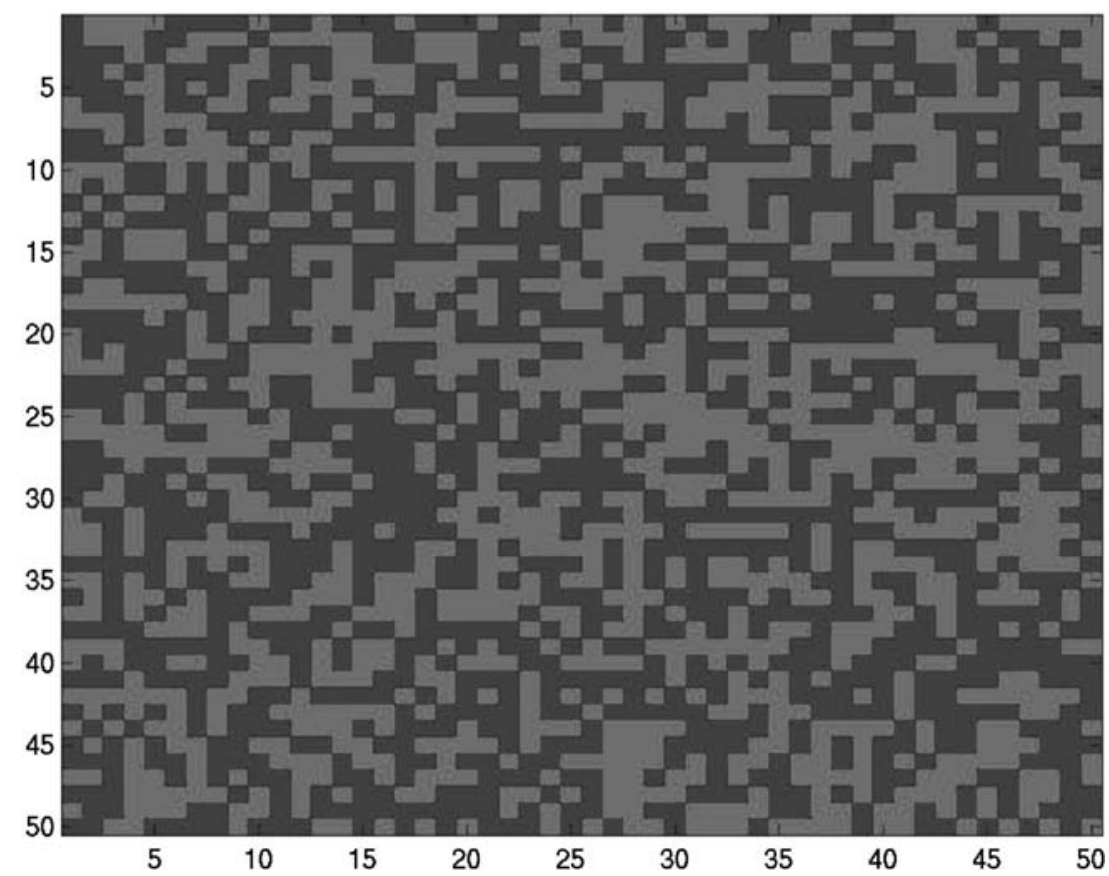

Figure 1. A $50 \times 50$ grid $($ red cells $=+1$, blue cells $=-1)$.

randomness is introduced into the model to account for an individual's changing her mind free of the influence of her neighbors. (A magnetic dipole always has some probability of flipping from spin up to spin down, even if all of its neighbors are spin up.) The probability of such a flip-flop is small but non-zero. In this simulation it is 0.001 .

What is observed is that if the initial distribution is evenly split between blue and red (i.e., $p=0.5$ ), then it tends to remain so for a considerable period of time (usually several thousand iterations for the grid sizes with which the simulation is concerned, $N=20$ or 30 , which one could call "the foreseeable future"). Even when $p$ is increased, neither side dominates until a considerable period of time has elapsed. But there is a critical threshold value of $p$ beyond which, even though $p$ may not be high, the density of blues increases to a high level, to the $90 \%$ range or higher.

Figure 4 gives the results in more detail. For grid sizes such as $N=20$ or $N=30$, the simulation was run 40 times with different initial densities $p$ of blue cells. The graphs represent averages over the 40 runs for fixed $p$ and $N$. The initial distribution was recorded (exactly $p N^{2}$ blue cells, rounded down to the nearest integer) at time $t=0$, and then the distribution after 1,000 steps. In the deterministic setting, represented by the first graph, populations are completely stable very early on. For reasons discussed later, the populations are not completely stable in the quantum setting; it appears as if the inevitable end-result is that blue will dominate totally. Surprisingly, the graphs obtained seem to be independent of the grid size $N$.

One notes that for $p=0.5$, neither blue nor red dominates the society in the shortor medium-term future. (If one side dominates in the long-term, it is only because of random fluctuations that may, for instance, take $p$ above the critical density. Eventually, the minority color will die, because the quantum fluctuations eat away at the edges of 


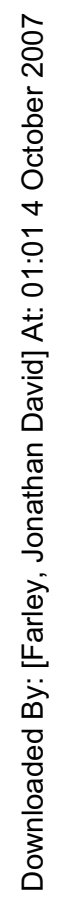

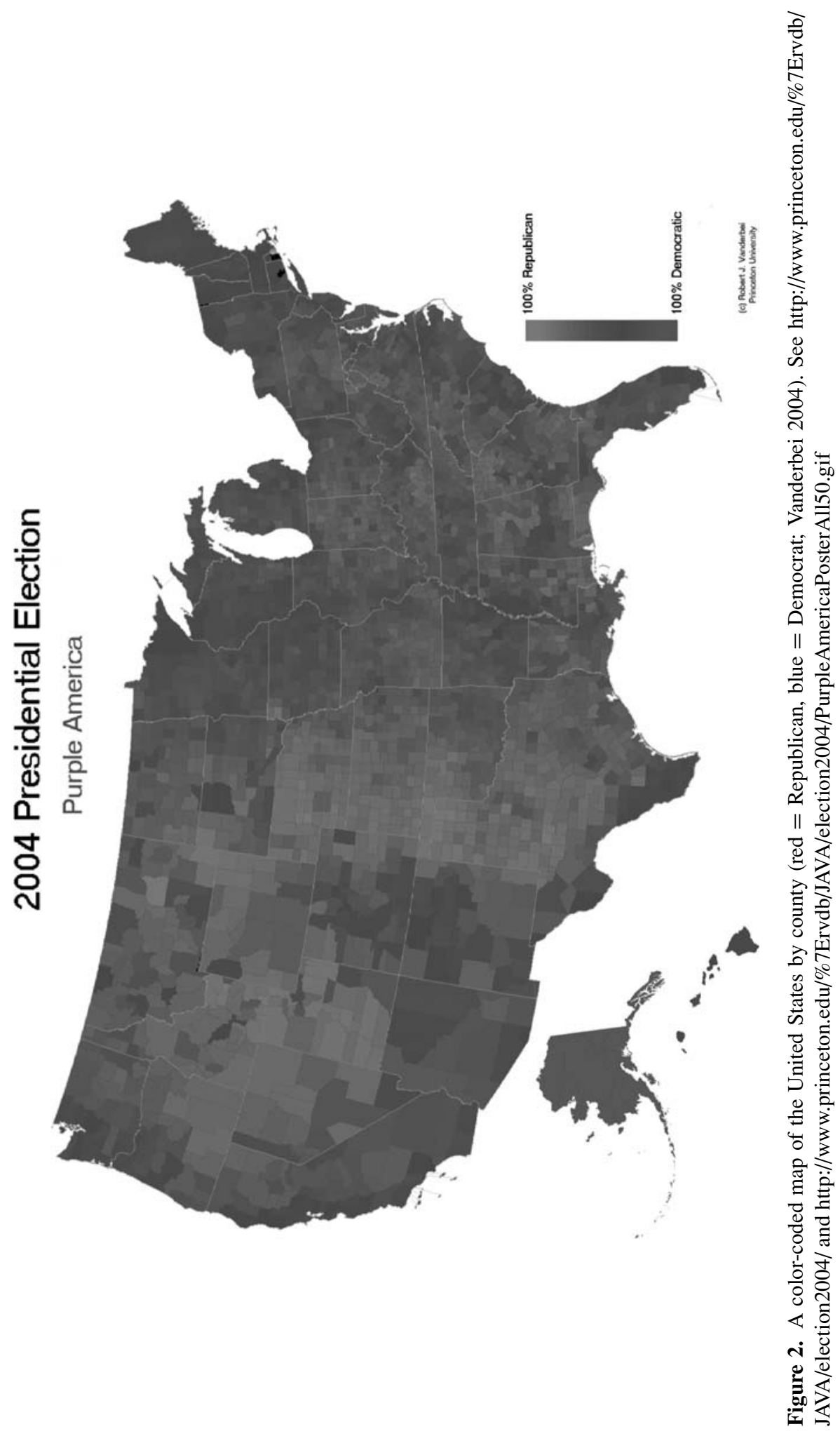




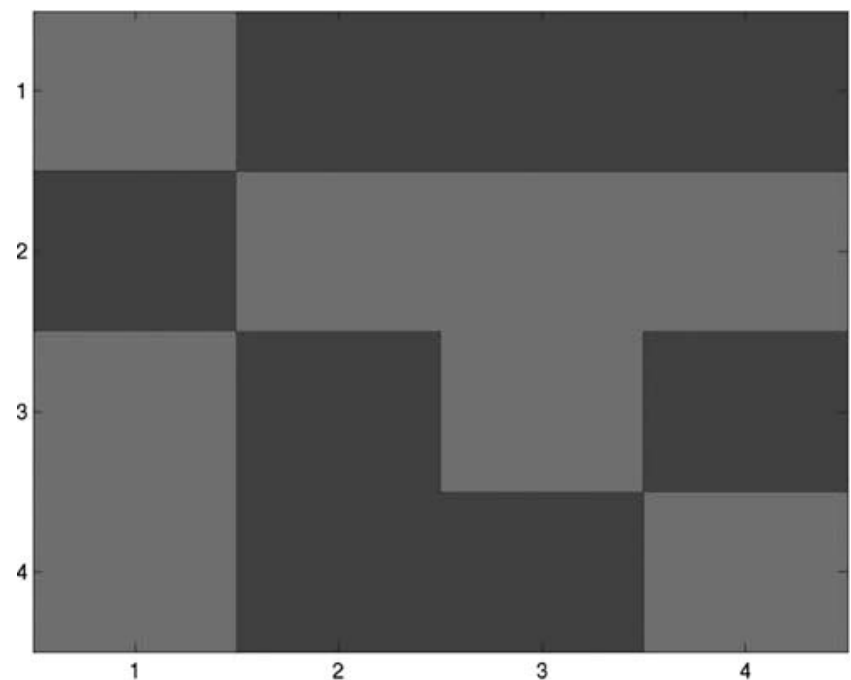

(a)

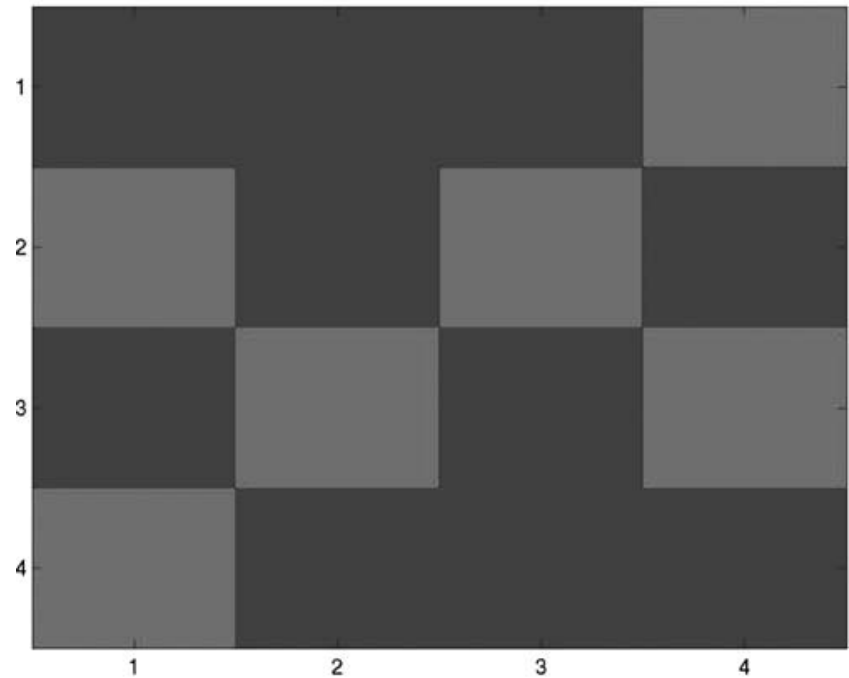

(b)

Figure 3. (a) A $4 \times 4$ grid. (b). The same $4 \times 4$ grid after one time-step.

that color's islands.) But above the critical density ( $p>0.55$ in the $20 \times 20$ case), blue's population very rapidly rises to close to $90 \%$ within 1,000 iterations, whereas even just a fraction below this threshold, blue does not gain so much so quickly and still must contend with a significant number of reds into the medium- and long-term future. This is a type 2 phase transition (Wolfram 2002).

Note that the critical density only applies when one has a random initial distribution. Once the simulation runs, structure emerges in the society (for example, islands or strips of red cells) that would resist elimination but for the quantum fluctuations. (For example, a $2 \times 2$ square of red cells would never disappear in the deterministic case.) Thus, blue 
may increase its percentage of the population to $70 \%$ and still not totally dominate red for thousands of iterations, even though the critical density may be well below 0.7 .

\section{Shortcomings of the Model and Some Generalizations}

A justification of the merits of running computer experiments has been given elsewhere (e.g., Bak and Chen 1991, 46; Johnson 1999; Lustick, Miodownik, and Eidelson 2004, 212; Orbell, Morikawa, Hartwig, Hanley, and Allen 2004, 3; Srbljinovic, Penzar, Rodik, and Kardov 2003, 1.4). The merits of the agent-based modeling approach may be found in Macy and Willer (2002); for the use of cellular automata specifically, see Hegselmann and Flache (1998). For a mathematical approach to the formation of public opinion using differential equations, see Abelson (1964); for one using matrices, see Harary (1959); for one using directed graphs, see French (1956). For a sample of other work using physical models for social phenomena, see Kenrick, Li, and Butner $(2003,14)$ and Lewenstein, Nowak, and Latané (1992). A good summary of other works discussing phase transitions on networks can be found in Newman (2003, section 8.4). For an example of using computers to model conflict, see Cederman (2003).

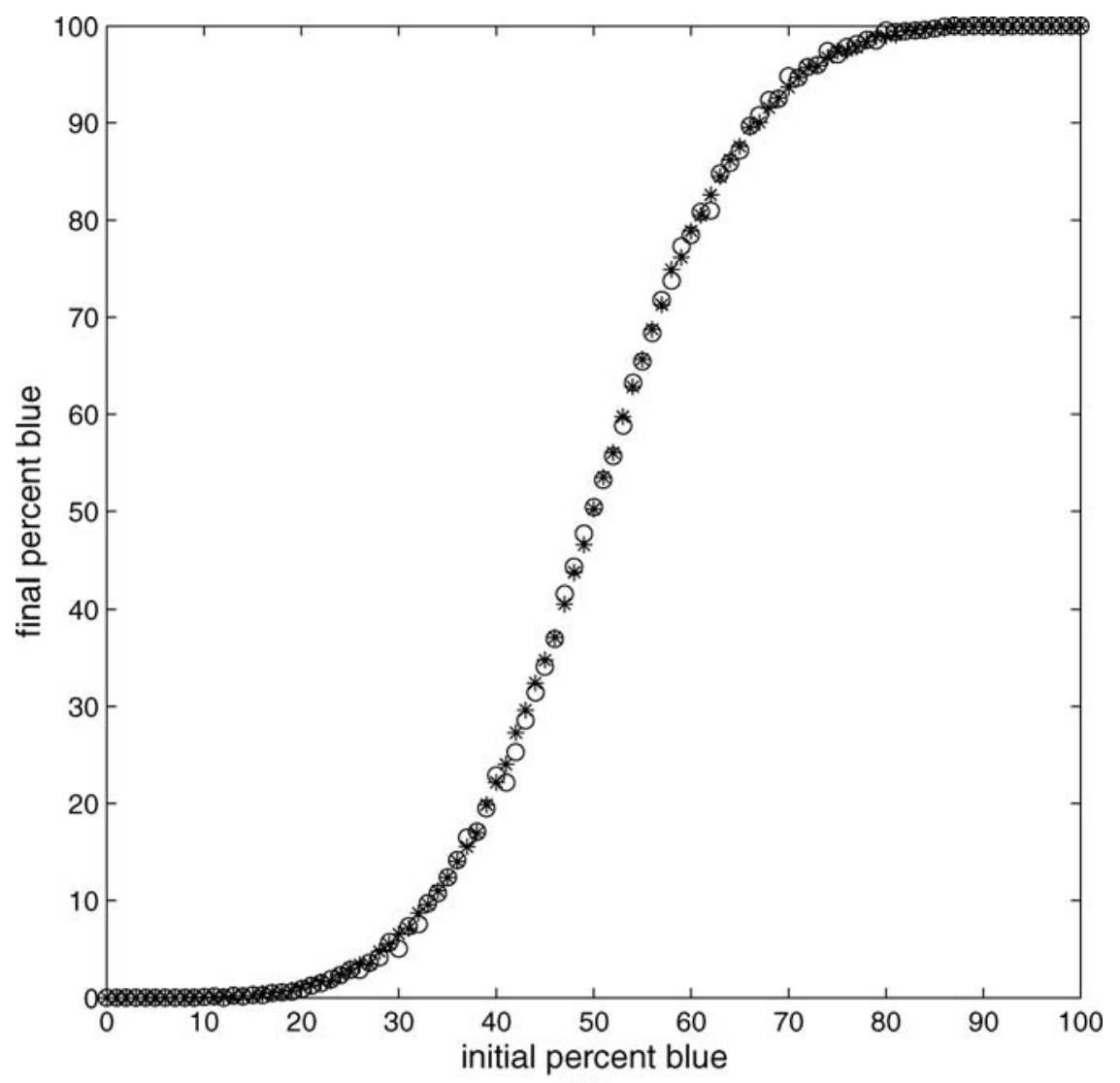

(a)

Figure 4. Graphs of the data after $t=1,000$ steps. Dynamic evolution of the automaton with and without quantum fluctuations. (Continued) 


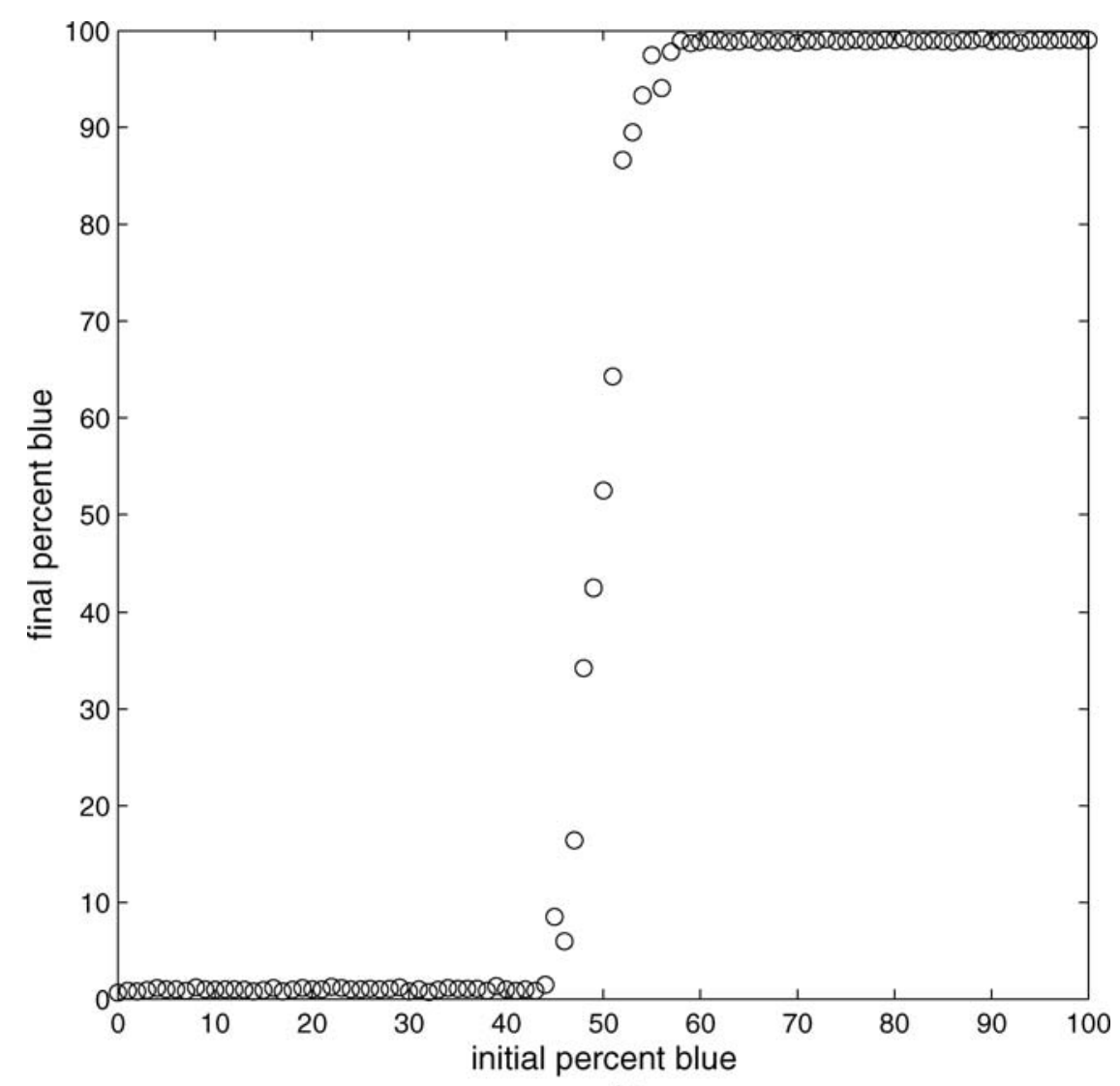

(b)

Figure 4. (Continued)

There are some interesting mathematical questions pertaining to this specific model. First, it would be interesting to prove analytically that the critical density exists and to calculate it. It would also be interesting to discover the function that gives the expected percentage of blues as a function of $t$ for fixed $p$ and $N$. Another interesting mathematical question is the behavior of islands. Because of the quantum fluctuation, it appears that islands inevitably decay (much as is the case with black holes under real quantum fluctuation; e.g., Schwarz 2005). What is this rate of decay? These questions of course depend on the specific construction of the model, but there are similar questions that might apply to more general models that would be equally, if not more, interesting.

One of the most obvious critiques of the model is its simplicity. The model certainly lacks the complexity of other simulations, for instance, those found in the works mentioned earlier. This weakness is only apparent. The model obviously recalls the housing segregation model of Schelling in the 1970s in which he demonstrates with a simple rule set that segregated housing patterns can emerge even under high levels of tolerance (Schelling 1971). It is well known that simple dynamical rules can produce complex, even chaotic, behavior (Mandelbrot 1982; Wolfram 2002). As has been observed, when trying to come to grips with fundamental phenomena, at least qualitatively, simplicity can be an advantage (Macy and Willer 2002, 162). ${ }^{3}$ The use of quantum fluctuations enables one to model opinion 
change. Zaller attempts to explain why individuals change their opinions when confronted by pollsters in successive months. "The generally accepted conclusion... has been that response instability... predominantly represents some sort of chance variation" (Zaller 1992, 30-31). Zaller himself posits that people do not have fixed opinions; they respond to pollsters with answers that depend on what is "at the top of the head" (Zaller 1992, 49). ${ }^{4}$

It is not so much that the present model is an improvement over other agent-based models than it is that the approach is different. This model is attempting to isolate a phenomenon that may be independent of the specific set of rules applied.

In this regard, the choice of simple rules has two advantages. First, it enables one to focus on what is causing the phenomenon of interest without the obfuscation of other complicating variables. Second, it could perhaps serve as the foundational model for a series of generalizations: What is the most general type of graph that yields a phase transition? It is conceivable that the quality "graph $G+$ rule set $R$ have a phase transition" is preserved under small modifications (conceptually) to the graph and rule set. Thus, there may be a pathway to proving a general theorem about what graphs with what sets of rules possess phase transitions, a result that might be inaccessible if its discovery were attempted from the beginning. To draw an analogy with the Church-Turing thesis, one deduces that any type of computer can be simulated by a Turing machine by showing, for example, that a standard Turing machine with alphabet $\{0,1\}$ can simulate a Turing machine with any finite alphabet; that a standard Turing machine with a one-dimensional tape can simulate a Turing machine that works on a two-dimensional grid; that a standard Turing machine can simulate a non-deterministic Turing machine, and so on. (Turing 1936-1937). It may in fact be the case that only very light conditions are required of the graph and rule set in order to produce a phase transition, just as (to give another very loose analogy) Arrow's impossibility theorem (Arrow 1951) or Gödel's incompleteness theorems (Gödel 1931) only require very reasonable conditions on the objects of interest (voting systems and axiom schemes for mathematics, respectively). The goal is ultimately to produce a theorem that could handle a much more complex system.

There are many directions in which one could expand the model. The most obvious one is to change the graph (see Flache and Hegselmann 2001; Kacperski and Holyst 2000). To better approximate real societies, one could see what results one gets with small-world networks or random graphs (Watts and Strogatz 1998; Bollobás 2001). Also, a more realistic way to handle islands would be to increase their resistance as their area shrinks (e.g., the Short Strand in Belfast). One could change the number of neighbors (for instance, one could use all eight neighbors instead of just the four to the north, south, east, and west; or one could create a hexagonal grid). One could add edges between cells that in the basic model are distance two apart (to model the fact that one's influence does not merely end with one's neighbors, and that the set of one's neighbors itself has a structure); in this case, one may add a kind of "inverse square" law, limiting the influence one has on these distance-two neighbors. (Such ideas formed the basis of the influence rules of Nowak et al. 1990.) One could introduce weightings across the board, for example, by using +1 for red and -0.5 for blue, or by giving one's own opinion extra weight when forming one's opinion in the next time interval. ${ }^{5}$ In real societies, not all opinions have equal weight; there are opinion leaders interspersed throughout the population (Green et al. 2004; Siegel 2005). There is also no reason why opinions must only be along one dimension; individuals could have opinions on multiple issues or there could be multiple possible opinions for any given issue.

Along similar lines, one could model the effect of propaganda, viewed as a magnetic field (so that cells tend toward the -1 opinion, for instance), or as a periodic pulse (every 10 time steps, for instance, to correspond to the news cycle). 
One could also analyze the effect on the model of introducing random long-distance connections, as when, for instance, one meets the acquaintance of an acquaintance in a far-away city. One could also more directly model mobility (cf. Sakoda 1971). One could also study the effect of barriers on influence propagation (e.g., oceans). The connection with the "critical mass" theory of collective action (Granovetter 1978) and the notion of "self-organized criticality" (Bak and Chen 1991) also deserve attention. There may also be fruitful applications to the understanding of the process by which juries reach a consensus (Tanford and Penrod 1983).

It would be interesting to understand how the present model deviates from the real world, in an attempt to see why phase transitions do not occur; or, if they have occurred in certain historical situations, to find them. Alternatively, one could try to create artificial environments in which the rules of the model do apply to see if the simulated results occur in reality. This might be done along the same lines as sleep experiments, in which 100 individuals (for example) are kept secluded in separate rooms and can only communicate with their neighbors.

\section{Occupational Hazards: Implications for Iraq}

Despite the shortcomings and primitive nature of the present model, there seem to be a number of potential applications. Networks in the real world will not exhibit the regularity and structure of the torus presently used. But perhaps actual networks-for example networks consisting of different television markets - could be studied so that groups wishing to influence public opinion (e.g., campaigns for political candidates) need not spend their resources trying to change every node in the network, but only a fraction of the nodes. This could represent a considerable savings for the group in question (provided, of course, that the topology of the appropriate influence networks was understood). Another application might be to mathematical epidemiology and virus propagation: +1 indicates that an individual has a cold, -1 indicates she does not. If a majority of your neighbors have colds, you are more likely to get a cold; otherwise, your body's natural defenses will enable you to stay healthy or recover from your cold, at least for one time step.

One could also model a situation where, for instance, negative views or rumors are spread throughout a population. For example, one could model the graph representing familial relationships in Iraqi society, and see what density of the population must experience an untoward event at the hands of occupation forces before the entire population has a negative view of the occupation (the initial -1 nodes being, for example, prisoners at Abu Ghraib).

On 1 May 2003, U.S. President George W. Bush declared an end to "major combat operations" in Iraq. It was clear, however, that the battle for the hearts and minds of the Iraqi people had only just begun. If the present hypotheses apply in some form to human societies, the results suggest that seemingly low percentages of antipathy toward the U.S. occupation forces could tip dramatically and without warning in favor of the insurgency. It therefore behooves policymakers to pay closer attention to public attitudes, even when it seems as if there is little cause for alarm.

\section{Outlook: A Theory of Everyone?}

Using a simple analogy from statistical physics, one can see what may be a general phenomenon about the spread of political influence and the formation of public opinion. 
Determining how far this model deviates from reality would be as worthwhile an area of investigation as determining how much the properties of the model carry over to other networks, and whether historical or laboratory examples could be found in the realm of human interactions.

Simple mathematics can be used to elucidate the fundamental interactions between atoms. Although one should not and must not look for a "theory of everyone," one also should not balk at using simple mathematics to try to elucidate some aspects of collective social behavior. The language of mathematics merely enables someone to state his or her assumptions and conclusions, and to present his or her reasoning, clearly and unambiguously. In the case of Iraq, it is important for policymakers and military commanders to know that the difference between mission accomplished and mission impossible may be as narrow as the knife's edge.

\section{PART II: EVOLUTIONARY DYNAMICS OF PUBLIC OPINION}

\section{Maximum Territorial Control in the Battle for Hearts and Minds}

This section applies the general considerations of Part I to a specific optimization problem. How does one convert a maximum amount of territory to one's side with constrained resources? Suppose a political party or a military commander is presented with the following situation: She is trying to gain mastery over a region. This region has $n$ loci of control (in the example that follows, there are five important cities in a country); each of these loci will enable the commander's forces to exert influence over a certain region surrounding it, her influence dwindling as the distance from that locus of power grows. The commander has the resources only to take over $k$ of the $n$ loci. (The exact number may depend on which loci are taken; see the possible expansions of the model in what follows.) The other $n-k$ will remain hostile. Which $k$ should the commander concentrate her resources on in order to maximize the amount of territory under her control?

\section{The Simulation}

There is a $N \times N$ grid of cells, that is, $N^{2}$ total cells. Initialize all of the grid cells to zero. A number of special red "source" cells are maintained at +1 throughout the simulation, and another number of blue source cells are maintained at -1 . The rule to update a cell is to take the average of a cell with its nearest horizontal and vertical neighbors:

$$
P_{i, j}^{n+1}=\frac{P_{i, j}^{n}+P_{i+1, j}^{n}+P_{i-1, j}^{n}+P_{i, j+1}^{n}+P_{i, j-1}^{n}}{5}
$$

When the computed average for a cell is greater than zero, that cell is colored a shade of red; if it is less than zero, it is colored a shade of blue. White cells indicate the transition from red to blue regions. A simulation is run until an equilibrium is reached.

\section{Results and Discussion}

In the specific example, there is a $20 \times 20$ grid of cells, that is, $N=20$. There are also five source cells, any three of which can be chosen as blue sources $(-1 \mathrm{~s})$, and the other two are red sources $(+1 \mathrm{ls})$. All ten possible configurations of three blue sources and two red sources 

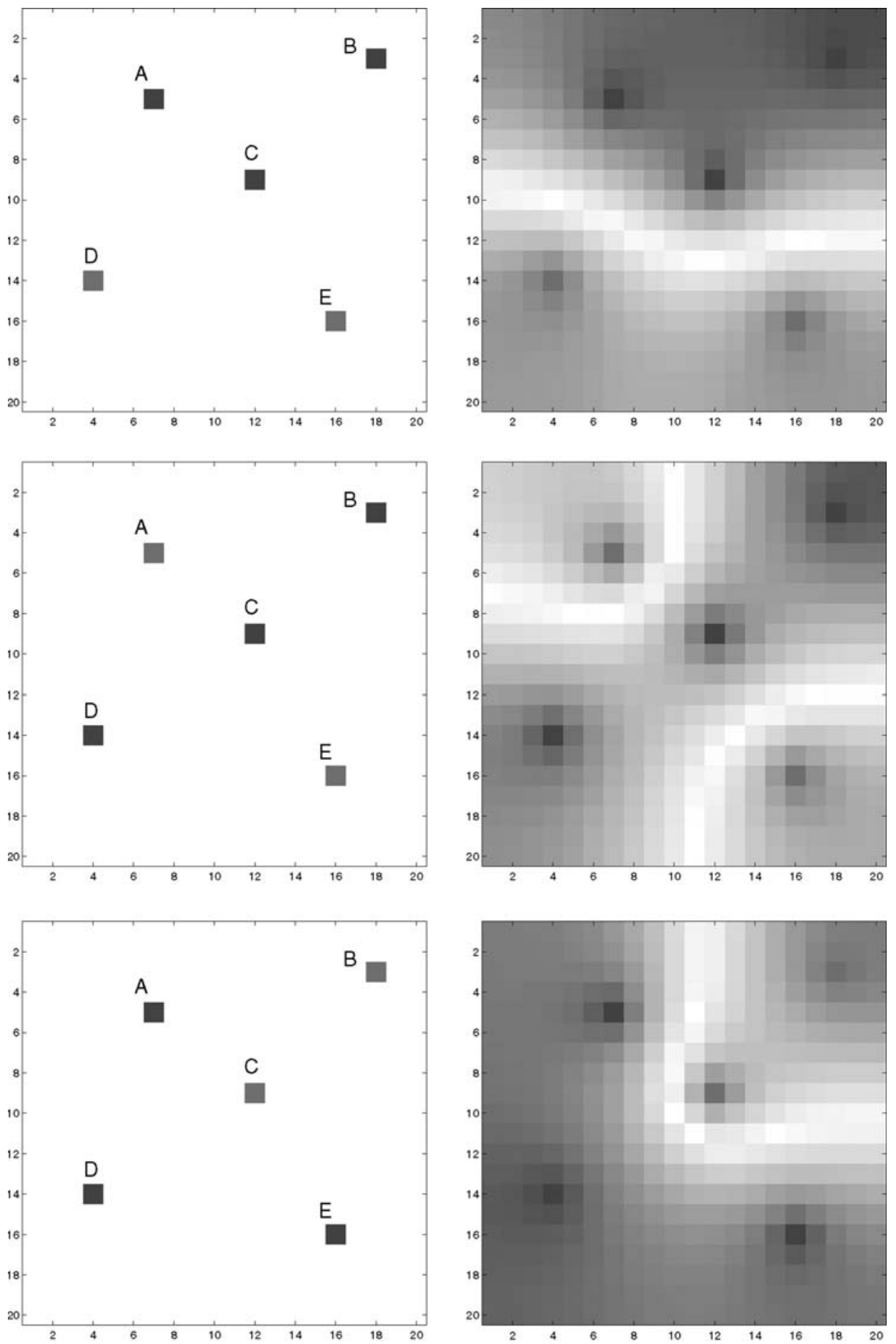

Figure 5. The diffusion of public opinion. 
Table 1

Maximum territorial control in the battle for hearts and minds

\begin{tabular}{lcccccc}
\hline Blue & Red & $\begin{array}{c}\text { Blue } \\
\text { territory }\end{array}$ & $\begin{array}{c}\text { Red } \\
\text { territory }\end{array}$ & $\begin{array}{c}\text { Blue } \\
\text { opinion }\end{array}$ & $\begin{array}{c}\text { Red } \\
\text { opinion }\end{array}$ & Time \\
\hline ABC & DE & 54.5 & 45.5 & 29.0 & 18.1 & 432 \\
ABD & CE & 64.2 & 35.8 & 29.8 & 12.6 & 377 \\
ABE & CD & 64.5 & 35.5 & 25.8 & 10.0 & 720 \\
ACD & BE & 66.2 & 33.8 & 37.0 & 11.5 & 561 \\
ACE & BD & 73.8 & 26.2 & 31.6 & 7.6 & 628 \\
ADE & BC & 74.5 & 25.5 & 39.3 & 8.9 & 842 \\
BCD & AE & 65.5 & 34.5 & 24.0 & 9.2 & 857 \\
BCE & AD & 57.0 & 43.0 & 30.8 & 17.4 & 146 \\
BDE & AC & 67.8 & 32.2 & 30.1 & 10.5 & 752 \\
CDE & AB & 68.0 & 32.0 & 38.6 & 10.7 & 912 \\
\hline
\end{tabular}

are run, and the results tabulated (Figure 5). The metrics that are tabulated are

$$
\text { blue territory }=\frac{\text { number of blue cells }}{\text { total number of cells }}
$$

and

$$
\text { blue opinion }=\frac{1}{N^{2}} \sum_{(i, j)=\text { blue }}\left|P_{i, j}\right|
$$

These values are given as percentages in Table 1. Also given is the time to stabilization, which is that time after which applying the rule does not change the value of any grid cell (up to the precision of the computer).

\section{Implications for Winning Hearts and Minds}

In a real military scenario, ground must not only be taken, it must be kept. Resources being finite (e.g., the U.S. troops were, according to reports, spread thin during the initial phases of the 2003 Iraq War), a commander may wish to focus her attention on specific spots. In an occupation, it is natural to assume that the regions not under the influence of the occupier will remain hostile. The commander wishes to maximize the area under her control. Where will the greatest payoff be? The same results would apply to a contest between two political parties in a region. In this interpretation, the model might lead to the "optimal" way to gerrymander political turf.

\section{Territory vs. Hearts and Minds}

More realistic models would include (at least) the following refinements: Control of area is different from attaining victory in "the battle for hearts and minds" (e.g., control of vast territory in the Soviet Union by German forces during World War II; the size of Tamerlane's empire; etc.). Defining "territorial control" by counting the number of blue squares therefore represents a "with us or against us" philosophy. The "degree of blueness" more accurately represents the degree to which the battle for hearts and minds has been 
won. The model incorporates this by summing over fractional amounts of blueness. Perhaps there are non-trivial configurations for which this quantity depends only on the number $k$ of occupied loci, not their location.

\section{Modeling Resistance and Resources}

A very simple, but very necessary, modification is to weight each locus by the difficulty of taking it (i.e., Fallujah vs. Kirkuk in Kurdistan). One suggestion is to choose weights based on population. (Defining a unit of area by population, another option, would be similar to the way congressional districts are defined in the United States.) Conversely, certain regions might be more highly valued-for instance, if they contained natural resources-so one might wish to weight squares according to their value.

\section{Boundary Effects and the Line of Control}

Another improvement would be to take into account boundary effects. Real regions have neighbors. (In Iraq, there might be a constant source of "redness" on the border with Iran.) Along these lines, it would be interesting to see if it is somehow "worse" to choose corner points.

\section{Simplicity Theory}

Along these lines, another "boundary" is the white line that separates the blue and red zones of control. It would be interesting to be able to analytically describe this "Mason-Dixon Line" (i.e., without having to run the simulation). It would also be interesting to be able to analytically describe the length of time it takes until the equilibrium is reached.

The ultimate question, of course, is if it is possible to analytically describe the best configuration, just given the locations of the $n$ loci and the number $k$. This leads to a set of questions that, on the surface, seem to fall into the realm of complexity theory. The key difference, however, is that in complexity theory one is confronted with problems for large values of $n$, whereas, in the present case, $n$ is unlikely to be more than 50 (and possibly no more than 5, as in the example). Thus "exponential time" algorithms might yield the optimal configuration in a feasible amount of time; conversely, known "polynomial time" algorithms could conceivably be too time-consuming, if they require vast amounts of time for overhead, or if the degree of the polynomial is too large.

\section{Scale-Freeness}

Finally, we note that the present simulation is scale-free in the sense that the same model would apply if one wanted to determine the best cities in a country to take over, or the best districts within a city (for instance, the optimal mosques to try to sway in order to have influence over the largest amount of territory within a city that is crucial, but still largely hostile).

\section{The Diffusion of Public Opinion}

The diffusion of heat in an object is a well-known and well-studied phenomenon. In mathematical physics, the diffusion of heat over time is described by the diffusion equation

$$
\frac{\partial T}{\partial t}=\nabla^{2} T
$$


where $T(x, y, z, t)$ is the temperature of an object as a function of space and time, and $\nabla^{2}$ is the Laplacian operator. The Laplacian in two dimensions is given by

$$
\nabla^{2} T=\frac{\partial^{2} T}{\partial x^{2}}+\frac{\partial^{2} T}{\partial y^{2}}
$$

This article discretizes the diffusion equation, using what is called a backward Euler for the time derivative and a center difference for the Laplace operator. (The author thanks a reader of this manuscript for these comments and the references Tikhonov and Samarskii 1963 and Strikwerda 1989.)

$$
\frac{T_{i, j}^{n+1}-T_{i, j}^{n}}{\Delta t}=\frac{T_{i+1, j}^{n}-2 T_{i, j}^{n}+T_{i-1, j}^{n}}{\Delta x^{2}}+\frac{T_{i, j+1}^{n}-2 T_{i, j}^{n}+T_{i, j-1}^{n}}{\Delta x^{2}}
$$

Rearranging, one has

$$
T_{i, j}^{n+1}=T_{i, j}^{n}+\frac{\Delta t}{\Delta x^{2}}\left[T_{i+1, j}^{n}+T_{i-1, j}^{n}+T_{i, j+1}^{n}+T_{i, j-1}^{n}-4 T_{i, j}^{n}\right]
$$

When $\Delta \mathrm{t} / \Delta x^{2}=1 / 5$, this becomes

$$
T_{i, j}^{n+1}=\frac{T_{i, j}^{n}+T_{i+1, j}^{n}+T_{i-1, j}^{n}+T_{i, j+1}^{n}+T_{i, j-1}^{n}}{5}
$$

This is the averaging rule used for the present simulations. Hence, one could argue by analogy that the simulations being performed are modeling the diffusion of public opinion. Note, however, that some caution needs to be exercised when discretizing differential equations (Durrett and Levin 1994).

\section{Notes}

1. Nor is the author asserting that phase transitions do not occur.

2. Individuals are often influenced by their neighbors, if all the residents on a street wish to use the same garbage collection company, for instance, or sell their land to a developer (who will pay more if he can get all of the properties at once). There is also the intimidation effect: an antiwar Democrat may feel it is unsafe to show his colors if everyone on his street has an SUV with a "support the troops" ribbon. Some of the ideas from social psychology on proximity and socialization could also be brought to bear. The proximity-attraction principle, that one is more likely to like familiar people, could be stretched to suggest that one is more likely to be like familiar people, that is, his or her neighbors. If the blues and reds are interpreted as being distinct political parties, further justification comes from Green, Schickler, and Palmquist (2004), chapter 1: "party identification ... is often acquired early in life as a product of one's family environment. ... [P] artisan identities ... also change as regional and occupational mobility put adults into contact with new friends and social groups, some of which may have different partisan coloration." Our rule also mirrors Zaller's Axiom A4, the "Response Axiom": "Individuals answer survey questions by averaging across the considerations that are immediately salient or accessible to them" (Zaller 1992, 49).

3. " $[\mathrm{I}] \mathrm{f}$ the goal is to deepen our understanding of some fundamental process, then simplicity of the assumptions is important and realistic representation of all the details of a particular setting is not." (Axelrod 1997, 25, quoted in Macy and Willer 2002, 147.)

4. The present author's view is that this corresponds to the "hidden variables" notion in quantum theory, contrasting with the notion that states of physical systems are only indeterminate waves capable 
of taking on many different values (the states become definite only when an observation is made and the wave function "collapses").

5. There has been considerable empirical evidence showing, for instance, that most individuals are holders of "non-opinions" and "non-attitudes" (Zaller 1992). According to Converse, "[L]arge portions of the electorate do not have meaningful beliefs" (Converse 1964, 245). These portions might be represented as 0 s.

\section{References}

Abelson, Robert P. 1964. Mathematical models of the distribution of attitudes under controversy. In Contributions to mathematical psychology, eds. Norman Frederiksen and Harold Gulliksen, 141-160. New York: Holt, Rinehart and Winston.

Arrow, Kenneth J. 1951. Social choice and individual values. New York: John Wiley \& Sons, Inc. Axelrod, R. 1997. The complexity of cooperation. Princeton, NJ: Princeton University Press. Bak, Per, and Kan Chen. 1991. Self-organized criticality. Scientific American 264 (January):46-53. Bollobás, Béla. 2001. Random graphs. 2nd ed. Cambridge: Cambridge University Press.

Cederman, Lars-Erik. 2003. Modeling the size of wars: From billiard balls to sandpiles. American Political Science Review 97:135-150.

Cipra, Barry A. 1987. An introduction to the Ising model. The American Mathematical Monthly 94 (December):937-959.

Converse, Philip. 1964. The nature of belief systems in mass publics. In Ideology and discontent, ed. David E. Apter, 206-261. New York: The Free Press of Glencoe.

Durrett, Richard, and Simon Levin. 1994. The importance of being discrete (and spatial). Theoretical Population Biology 46:363-394.

Flache, Andreas, and Rainer Hegselmann. 2001. Do irregular grids make a difference? Relaxing the spatial regularity assumption in cellular models of social dynamics. Journal of Artificial Societies and Social Simulation 4 (October:) (http://www.soc.surrey.ac.uk/JASSS/4/4/6.html)

French, John R. P. 1956. A formal theory of social power. Psychological Review 63:181-194.

Gödel, K. 1931. Über formal unentscheidbare Sätze der Principia Mathematica und verwandter Systeme, I. Monatschefte für Mathematik und Physik 38:173-198.

Granovetter, Mark 1978. Threshold models of collective behavior. American Journal of Sociology 83 (May):1420-1443.

Green, Donald P., Eric Schickler, and Bradley Palmquist. 2004. Partisan hearts and minds: Political parties and the social identities of voters. New Haven, CT: Yale University Press.

Harary, Frank. 1959. A criterion for unanimity in French's theory of social power. In Studies in Social Power, ed. Dorwin Cartwright, 168-182. Ann Arbor: The University of Michigan Press.

Hegselmann, Rainer, and Andreas Flache. 1998. Understanding complex social dynamics: A plea for cellular automata based modelling. Journal of Artificial Societies and Social Simulation 1 (June) (http://www.soc.surrey.ac.uk/JASSS/1/3/1.html)

Ising, Ernst. 1925. Beitrag zur theorie des ferromagnetismus. Zeitschrift für Physik 31:253-258.

Johnson, P. E. 1999. Simulation modeling in political science. American Behavioral Scientist 42:1509-1530.

Kacperski, Krzysztof, and Janusz A. Holyst. 2000. Phase transitions as a persistent feature of groups with leaders in models of opinion formation. Physica A 287:631-643.

Kenrick, Douglas T., Norman P. Li, and Jonathan Butner. 2003. Dynamical evolutionary psychology: Individual decision rules and emergent social norms. Psychological Review 110:3-28.

Krepinevich, Andrew F. 2005. How to win in Iraq. Foreign Affairs 84.

Lewenstein, Maciej, Andrzej Nowak, and Bibb Latané. 1992. Statistical mechanics of social impact. Physical Review A 45:763-776.

Lustick, Ian S., Dan Miodownik, and Roy J. Eidelson. 2004. Secessionism in multicultural states: Does sharing power prevent or encourage it? American Political Science Review 98:209-229. 
Macy, Michael W., and Robert Willer. 2002. From factors to actors: Computational sociology and agent-based modeling. Annual Review of Sociology 28:143-166.

Mandelbrot, Benoit B. 1982. The fractal geometry of nature. San Francisco: W. H. Freeman and Co.

Newman, M. E. J. 2003. The structure and function of complex networks. SIAM Review 45:167-256.

Nowak, Andrzej, Jacek Szamrej, and Bibb Latané. 1990. From private attitude to public opinion: A dynamic theory of social impact. Psychological Review 97:362-376.

Orbell, John, Tomonori Morikawa, Jason Hartwig, James Hanley, and Nicholas Allen. 2004. "Machiavellian" intelligence as a basis for the evolution of cooperative decisions. American Political Science Review 98:1-15.

Peierls, R. 1936. On Ising's model of ferromagnetism. Proceedings of the Cambridge Philosophical Society 32:477-481.

Sakoda, James M. 1971. The checkerboard model of social interaction. Journal of Mathematical Sociology 1:119-132.

Schelling, T. C. 1971. Dynamic models of segregation. Journal of Mathematical Sociology 1:143-186.

Schwarz, Patricia. 2005. Hawking radiation poses new questions. Available at (http:// superstringtheory.com/blackh/blackh3.html).

Siegel, David A. 2005. When does repression work? Collective behavior under the threat of violence. Unpublished manuscript.

Srbljinovic, Armano, Drazen Penzar, Petra Rodik, and Kruno Kardov. 2003. An agent-based model of ethnic mobilisation. Journal of Artificial Societies and Social Simulation 6 (January) (http://jasss.soc.surry.ac.uk/6/1/1.html)

Strikwerda, J. C. 1989. Finite difference schemes and partial differential equations. New York: Chapman and Hall.

Tanford, S., and S. Penrod. 1983. Computer modelling of influence in the jury: The role of the consistent juror. Social Psychology Quarterly 46:200-212.

Tikhonov, A. N., and A. A. Samarskii. 1963. Equations of mathematical physics. New York: Dover Publications, Inc.

Turing, A. M. 1936-1937. On computable numbers, with an application to the Entscheidungsproblem. Proceedings of the London Mathematical Society 42:230-265.

Vanderbei, Robert J. 2004. 2004 presidential election: Purple America. Available at (http://www.princeton.edu/\%7Ervdb/JAVA/election2004/PurpleAmericaPosterAll50.gif).

Von Neumann, John. 1966. Theory of self-reproducing automata. Urbana: University of Illinois Press.

Watts, Duncan J., and Steven H. Strogatz. 1998. Collective dynamics of "small-world" networks. Nature 393 (June):440-442.

Wolfram, Stephen. 2002. A new kind of science. Champaign, IL: Wolfram Media, Inc.

Young, Ronald. 2004. An institute for mathematical methods in counterterrorism? A new model for north-south cooperation. Presented at the DIMACS Workshop on Applications of Order Theory to Homeland Defense and Computer Security.

Zaller, John R. 1992. The nature and origins of mass opinion. Cambridge: Cambridge University Press. 